\title{
Strong Differential Subordination Results for Multivalent Analytic Functions Associated with Dziok-Srivastava Operator
}

\author{
Abbas Kareem Wanas ${ }^{1}$ and Hala Abbas Mehdi ${ }^{2}$ \\ ${ }^{1}$ Department of Mathematics, College of Science, University of Al-Qadisiyah, Iraq \\ e-mail: abbas.kareem.w@qu.edu.iq \\ ${ }^{2}$ Department of Mathematics, College of Computer Science and Information Technology, \\ University of Al-Qadisiyah, Iraq; e-mail: halahaider2015@gmail.com
}

\begin{abstract}
In this paper, by making use of the principle of strong subordination, we establish some interesting properties of multivalent analytic functions defined in the open unit disk and closed unit disk of the complex plane associated with Dziok-Srivastava operator.
\end{abstract}

\section{Introduction and Preliminaries}

Denote by $\mathcal{H}(U \times \bar{U})$ the family of all analytic functions in $U \times \bar{U}$. Let $U=\{z \in \mathbb{C}:|z|<1\}$ and $\bar{U}=\{z \in \mathbb{C}:|z| \leq 1\}$ indicate the open unit disk and the closed unit disk of the complex plane, respectively. For $n \in \mathbb{N}=\{1,2, \ldots\}$ and $a \in \mathbb{C}$, let $\mathcal{H}^{*}[a, n, \zeta]=\left\{f \in \mathcal{H}(U \times \bar{U}): f(z, \zeta)=a+a_{n}(\zeta) z^{n}+a_{n+1}(\zeta) z^{n+1}+\cdots, z \in U\right.$, $\zeta \in \bar{U}\}$, where $a_{k}(\zeta)$ are holomorphic functions in $\bar{U}$ for $k \geq n$.

Also, let $\mathcal{A}_{n \zeta}^{*}=\left\{f \in \mathcal{H}(U \times \bar{U}): f(z, \zeta)=z+a_{n+1}(\zeta) z^{n+1}+\cdots, z \in U, \zeta \in \bar{U}\right\}$, with $\mathcal{A}_{1 \zeta}^{*}=\mathcal{A}_{\zeta}^{*}$, where $a_{k}(\zeta)$ are holomorphic functions in $\bar{U}$ for $k \geq n+1$.

Received: April 5, 2019; Accepted: May 10, 2019

2010 Mathematics Subject Classification: 30C45, 30A20, 34A40.

Keywords and phrases: multivalent functions, convex function, strong differential subordinations, DziokSrivastava operator.

Copyright (C) 2019 Abbas Kareem Wanas and Hala Abbas Mehdi. This is an open access article distributed under the Creative Commons Attribution License, which permits unrestricted use, distribution, and reproduction in any medium, provided the original work is properly cited. 
A function $f \in \mathcal{H}^{*}[a, n, \zeta]$ is said to be starlike in $U \times \bar{U}$ if

$$
\operatorname{Re}\left\{\frac{z f_{z}^{\prime}(z, \zeta)}{f(z, \zeta)}\right\}>0, \quad(z \in U, \zeta \in \bar{U})
$$

Denote the class of all starlike functions in $U \times \bar{U}$ by $S_{\zeta}^{*}$.

Similar, $f \in \mathcal{H}^{*}[a, n, \zeta]$ is said to be convex in $U \times \bar{U}$ if

$$
\operatorname{Re}\left\{\frac{z f_{2}^{\prime \prime}(z, \zeta)}{f_{z}^{\prime}(z, \zeta)}+1\right\}>0, \quad(z \in U, \zeta \in \bar{U}) .
$$

Denote the class of all convex functions in $U \times \bar{U}$ by $K_{\zeta}^{*}$.

Definition 1.1 [9]. Let $f(z, \zeta), g(z, \zeta)$ be analytic in $U \times \bar{U}$. The function $f(z, \zeta)$ is said to be strongly subordinate to $g(z, \zeta)$, written $f(z, \zeta) \prec \prec g(z, \zeta)$, $z \in U, \quad \zeta \in \bar{U}$, if there exists an analytic function $w$ in $U$ with $w(0)=0$ and $|w(z)|<1, \quad z \in U$ such that $f(z, \zeta)=g(w(z), \zeta)$ for all $\zeta \in \bar{U}$.

Remark 1.1 [9].

(1) Since $f(z, \zeta)$ is analytic in $U \times \bar{U}$, for all $\zeta \in \bar{U}$ and univalent in $U$, for all $\zeta \in \bar{U}$, Definition 1.1 is equivalent to $f(0, \zeta)=g(0, \zeta)$ for all $\zeta \in \bar{U}$ and $f(U \times \bar{U})$ $\subset g(U \times \bar{U})$.

(2) If $f(z, \zeta)=f(z)$ and $g(z, \zeta)=g(z)$, then the strong subordination becomes the usual notion of subordination.

Let $\mathcal{A}_{\zeta}^{*}(p)$ denote the subclass of the functions $f(z, \zeta) \in \mathcal{H}(U \times \bar{U})$ of the form:

$$
f(z, \zeta)=z^{p}+\sum_{k=1}^{\infty} a_{p+k}(\zeta) z^{p+k}, \quad p \in \mathbb{N}=\{1,2, \ldots\}, z \in U, \zeta \in \bar{U}
$$

which are analytic and multivalent in $U \times \bar{U}$.

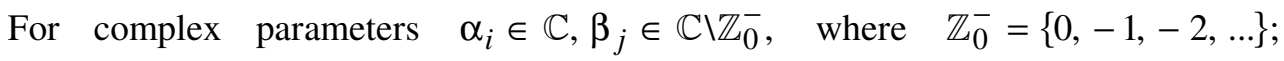
$1 \leq i \leq l, \quad 1 \leq j \leq m ; \quad l \leq m+1 ; \quad l, m \in \mathbb{N}_{0}=\mathbb{N} \cup\{0\}$ and $f \in \mathcal{A}_{\zeta}^{*}(p)$. The Dziok- 
Srivastava operator $H_{p}\left(\alpha_{1}, \ldots, \alpha_{l} ; \beta_{1}, \ldots, \beta_{m}\right): \mathcal{A}_{\zeta}^{*}(p) \rightarrow \mathcal{A}_{\zeta}^{*}(p)$ (see for details [3, 6]) is defined by

$$
H_{p}\left(\alpha_{1}, \ldots, \alpha_{l} ; \beta_{1}, \ldots, \beta_{m}\right) f(z, \zeta)=z^{p}+\sum_{k=1}^{\infty} \frac{\left(\alpha_{1}\right)_{k} \cdots\left(\alpha_{l}\right)_{k}}{\left(\beta_{1}\right)_{k} \cdots\left(\beta_{m}\right)_{k} k !} a_{p+k}(\zeta) z^{p+k},
$$

where $(x)_{k}$ is the Pochhammer symbol defined by

$$
(x)_{k}=\frac{\Gamma(x+k)}{\Gamma(x)}= \begin{cases}1 & (k=0), \\ x(x+1) \cdots(x+k-1) & (k \in \mathbb{N}) .\end{cases}
$$

In order to make the notation simple, we write $H_{p}^{l, m}\left(\alpha_{1}\right)=H_{p}\left(\alpha_{1}, \ldots, \alpha_{l} ; \beta_{1}, \ldots, \beta_{m}\right)$.

It is easily verified from (1.2) that

$$
z\left(H_{p}^{l, m}\left(\alpha_{1}\right) f(z, \zeta)\right)_{z}^{\prime}=\alpha_{1} H_{p}^{l, m}\left(\alpha_{1}+1\right) f(z, \zeta)-\left(\alpha_{1}-p\right) H_{p}^{l, m}\left(\alpha_{1}\right) f(z, \zeta) .
$$

We note that special cases of the Dziok-Srivastava operator $H_{p}^{l, m}\left(\alpha_{1}\right)$ include the Hohlov linear operator [4], the Carlson-Shafer operator [1], the Ruscheweyh derivative operator [11], the Srivastava-Owa fractional operator [10], and many others.

In recent years, many authors obtained various interesting results associated with strong differential subordination and superordination, for example, (see [2, 5, 12, 13, 14, 15]).

We will require the following lemmas in proving our main results:

Lemma 1.1 [8]. Let $h(z, \zeta)$ be a convex function with $h(0, \zeta)=a$, for every $\zeta \in \bar{U}$ and let $\gamma \in \mathbb{C}^{*}=\mathbb{C} \backslash\{0\}$ with $\operatorname{Re}(\gamma) \geq 0$. If $p \in \mathcal{H}^{*}[a, n, \zeta]$ and

$$
p(z, \zeta)+\frac{1}{\gamma} z p_{z}^{\prime}(z, \zeta) \prec \prec h(z, \zeta), \quad(z \in U, \zeta \in \bar{U}),
$$

then

$$
p(z, \zeta) \prec \prec q(z, \zeta) \prec \prec h(z, \zeta), \quad(z \in U, \zeta \in \bar{U}),
$$

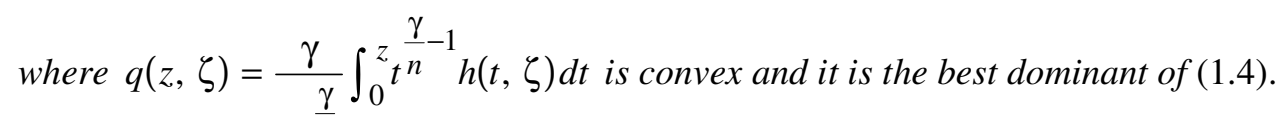

$$
n z^{n}
$$


Lemma 1.2 [7]. Let $q(z, \zeta)$ be a convex function in $U \times \bar{U}$ for all $\zeta \in \bar{U}$ and let $h(z, \zeta)=q(z, \zeta)+n \delta z q_{z}^{\prime}(z, \zeta), z \in U, \zeta \in \bar{U}$, where $\delta>0$ and $n$ is a positive integer. If

$$
p(z, \zeta)=q(0, \zeta)+p_{n}(\zeta) z^{n}+p_{n+1}(\zeta) z^{n+1}+\cdots,
$$

is analytic in $U \times \bar{U}$ and

$$
p(z, \zeta)+\delta z p_{z}^{\prime}(z, \zeta) \prec \prec h(z, \zeta), \quad(z \in U, \zeta \in \bar{U}),
$$

then

$$
p(z, \zeta) \prec \prec q(z, \zeta), \quad(z \in U, \zeta \in \bar{U}),
$$

and this result is sharp.

\section{Main Results}

Theorem 2.1. Let $h(z, \zeta)$ be a convex function such that $h(0, \zeta)=1$. If $f \in \mathcal{A}_{\zeta}^{*}(p)$ satisfies the strong differential subordination:

$$
\frac{\left(H_{p}^{l, m}\left(\alpha_{1}\right) f(z, \zeta)\right)_{z}^{\prime}}{p z^{p-1}} \prec \prec h(z, \zeta),
$$

then

$$
\frac{H_{p}^{l, m}\left(\alpha_{1}\right) f(z, \zeta)}{z^{p}} \prec \prec q(z, \zeta) \prec \prec h(z, \zeta),
$$

where $q(z, \zeta)=\frac{p}{z^{p}} \int_{0}^{z} t^{p-1} h(t, \zeta) d t$ is convex and it is the best dominant.

Proof. Suppose that

$$
F(z, \zeta)=\frac{H_{p}^{l, m}\left(\alpha_{1}\right) f(z, \zeta)}{z^{p}}, \quad z \in U, \quad \zeta \in \bar{U} .
$$

Then the function $F(z, \zeta)$ is analytic in $U \times \bar{U}$ and $F(0, \zeta)=1$. 
Simple computations from (2.2), we get

$$
F(z, \zeta)+\frac{1}{p} z F_{z}^{\prime}(z, \zeta)=\frac{\left(H_{p}^{l, m}\left(\alpha_{1}\right) f(z, \zeta)\right)_{z}^{\prime}}{p z^{p-1}} .
$$

Using (2.3), (2.1) becomes

$$
F(z, \zeta)+\frac{1}{p} z F_{z}^{\prime}(z, \zeta) \prec \prec h(z, \zeta)
$$

An application of Lemma 1.1 with $n=1, \gamma=p$ yields

$$
\frac{H_{p}^{l, m}\left(\alpha_{1}\right) f(z, \zeta)}{z^{p}} \prec \prec q(z, \zeta)=\frac{p}{z^{p}} \int_{0}^{z} t^{p-1} h(t, \zeta) d t \prec \prec h(z, \zeta) .
$$

By taking $p=1$ and $h(z, \zeta)=\frac{\zeta+(2 \lambda-\zeta) z}{1+z}, 0 \leq \lambda<1$ in Theorem 2.1, we obtain the following corollary:

Corollary 2.1. If $f \in \mathcal{A}_{\zeta}^{*}(1)$ satisfies the strong differential subordination:

$$
\left(H_{1}^{l, m}\left(\alpha_{1}\right) f(z, \zeta)\right)_{z}^{\prime} \prec \prec \frac{\zeta+(2 \lambda-\zeta) z}{1+z}
$$

then

$$
\frac{H_{1}^{l, m}\left(\alpha_{1}\right) f(z, \zeta)}{z} \prec \prec \frac{1}{z} \int_{0}^{z \zeta+(2 \lambda-\zeta) t} d t=2 \lambda-\zeta+\frac{2(\zeta-\lambda)}{z} \ln (1+z) .
$$

Theorem 2.2. Let $h(z, \zeta)$ be a convex function such that $h(0, \zeta)=1$. If $0 \leq \sigma<p$, $\eta \in \mathbb{C}$ and $f \in \mathcal{A}_{\zeta}^{*}(p)$ satisfies the strong differential subordination:

$$
\frac{1-\eta}{1-\sigma}\left(\frac{H_{p}^{l, m}\left(\alpha_{1}\right) f(z, \zeta)}{z^{p}}-\sigma\right)+\frac{\eta}{1-\sigma}\left(\frac{\left(H_{p}^{l, m}\left(\alpha_{1}\right) f(z, \zeta)\right)_{z}^{\prime}}{p z^{p-1}}-\sigma\right) \prec \prec h(z, \zeta),
$$

then

$$
\frac{1}{1-\sigma}\left(\frac{H_{p}^{l, m}\left(\alpha_{1}\right) f(z, \zeta)}{z^{p}}-\sigma\right) \prec \prec q(z, \zeta) \prec \prec h(z, \zeta)
$$


where $q(z, \zeta)=\frac{p}{\eta} z^{-\frac{p}{\eta}} \int_{0}^{z} t^{\frac{p}{\eta}-1} h(t, \zeta) d t$ is convex and it is the best dominant.

Proof. Suppose that

$$
F(z, \zeta)=\frac{1}{1-\sigma}\left(\frac{H_{p}^{l, m}\left(\alpha_{1}\right) f(z, \zeta)}{z^{p}}-\sigma\right), \quad z \in U, \quad \zeta \in \bar{U} .
$$

Then the function $F(z, \zeta)$ is analytic in $U \times \bar{U}$ and $F(0, \zeta)=1$.

Differentiating both sides of (2.5) with respect to $z$, we have

$$
\begin{aligned}
F(z, \zeta)+\frac{\eta}{p} z F_{z}^{\prime}(z, \zeta)= & \frac{1-\eta}{1-\sigma}\left(\frac{H_{p}^{l, m}\left(\alpha_{1}\right) f(z, \zeta)}{z^{p}}-\sigma\right) \\
& +\frac{\eta}{1-\sigma}\left(\frac{\left(H_{p}^{l, m}\left(\alpha_{1}\right) f(z, \zeta)\right)_{z}^{\prime}}{p z^{p-1}}-\sigma\right)
\end{aligned}
$$

From (2.4) and (2.6), we get

$$
F(z, \zeta)+\frac{\eta}{p} z F_{z}^{\prime}(z, \zeta) \prec \prec h(z, \zeta)
$$

An application of Lemma 1.1 with $n=1, \gamma=\frac{p}{\eta}$ yields

$$
\frac{1}{1-\sigma}\left(\frac{H_{p}^{l, m}\left(\alpha_{1}\right) f(z, \zeta)}{z^{p}}-\sigma\right) \prec \prec q(z, \zeta)=\frac{p}{\eta} z^{-\frac{p}{\eta}} \int_{0}^{z} t^{\frac{p}{\eta}-1} h(t, \zeta) d t \prec \prec h(z, \zeta) .
$$

Theorem 2.3. Let $q(z, \zeta)$ be a convex function such that $q(0, \zeta)=1$ and let $h$ be the function $h(z, \zeta)=q(z, \zeta)+z q_{z}^{\prime}(z, \zeta)$. If $f \in \mathcal{A}_{\zeta}^{*}(p)$ satisfies the strong differential subordination:

$$
\left(\frac{z H_{p}^{l, m}\left(\alpha_{1}+1\right) f(z, \zeta)}{H_{p}^{l, m}\left(\alpha_{1}\right) f(z, \zeta)}\right)_{z}^{\prime} \prec \prec h(z, \zeta),
$$


then

$$
\frac{H_{p}^{l, m}\left(\alpha_{1}+1\right) f(z, \zeta)}{H_{p}^{l, m}\left(\alpha_{1}\right) f(z, \zeta)} \prec \prec q(z, \zeta)
$$

Proof. Suppose that

$$
F(z, \zeta)=\frac{H_{p}^{l, m}\left(\alpha_{1}+1\right) f(z, \zeta)}{H_{p}^{l, m}\left(\alpha_{1}\right) f(z, \zeta)}, \quad z \in U, \quad \zeta \in \bar{U} .
$$

Then the function $F(z, \zeta)$ is analytic in $U \times \bar{U}$ and $F(0, \zeta)=1$.

Differentiating both sides of (2.8) with respect to $z$ and using (2.7), we have

$$
\begin{aligned}
& F(z, \zeta)+z F_{z}^{\prime}(z, \zeta) \\
= & \frac{H_{p}^{l, m}\left(\alpha_{1}+1\right) f(z, \zeta)}{H_{p}^{l, m}\left(\alpha_{1}\right) f(z, \zeta)} \\
+ & \frac{H_{p}^{l, m}\left(\alpha_{1}\right) f(z, \zeta)\left(H_{p}^{l, m}\left(\alpha_{1}+1\right) f(z, \zeta)\right)_{z}^{\prime}-H_{p}^{l, m}\left(\alpha_{1}+1\right) f(z, \zeta)\left(H_{p}^{l, m}\left(\alpha_{1}\right) f(z, \zeta)\right)_{z}^{\prime}}{\left[H_{p}^{l, m}\left(\alpha_{1}\right) f(z, \zeta)\right]^{2}} \\
= & \frac{H_{p}^{l, m}\left(\alpha_{1}\right) f(z, \zeta)\left(z H_{p}^{l, m}\left(\alpha_{1}+1\right) f(z, \zeta)\right)_{z}^{\prime}-z H_{p}^{l, m}\left(\alpha_{1}+1\right) f(z, \zeta)\left(H_{p}^{l, m}\left(\alpha_{1}\right) f(z, \zeta)\right)_{z}^{\prime}}{\left[H_{p}^{l, m}\left(\alpha_{1}\right) f(z, \zeta)\right]^{2}} \\
= & \left(\frac{z H_{p}^{l, m}\left(\alpha_{1}+1\right) f(z, \zeta)}{H_{p}^{l, m}\left(\alpha_{1}\right) f(z, \zeta)}\right)_{z}^{\prec \prec h(z, \zeta) .}
\end{aligned}
$$

An application of Lemma 1.2, we obtain

$$
\frac{H_{p}^{l, m}\left(\alpha_{1}+1\right) f(z, \zeta)}{H_{p}^{l, m}\left(\alpha_{1}\right) f(z, \zeta)} \prec \prec q(z, \zeta) .
$$

Theorem 2.4. Let $q(z, \zeta)$ be a convex function such that $q(0, \zeta)=1$ and let $h$ be the function $h(z, \zeta)=q(z, \zeta)+\frac{1}{\alpha_{1}+p} z q_{z}^{\prime}(z, \zeta)$, where $\alpha_{1}>0$. Suppose that 


$$
G(z, \zeta)=\frac{\alpha_{1}+p}{z^{\alpha_{1}}} \int_{0}^{z} t^{\alpha_{1}-1} f(t, \zeta) d t, \quad z \in U, \quad \zeta \in \bar{U}
$$

If $f \in \mathcal{A}_{\zeta}^{*}(p)$ satisfies the strong differential subordination

$$
\frac{\left(H_{p}^{l, m}\left(\alpha_{1}\right) f(z, \zeta)\right)_{z}^{\prime}}{p z^{p-1}} \prec \prec h(z, \zeta),
$$

then

$$
\frac{\left(H_{p}^{l, m}\left(\alpha_{1}\right) G(z, \zeta)\right)_{z}^{\prime}}{p z^{p-1}} \prec \prec(z, \zeta) .
$$

Proof. Suppose that

$$
F(z, \zeta)=\frac{\left(H_{p}^{l, m}\left(\alpha_{1}\right) G(z, \zeta)\right)_{z}^{\prime}}{p z^{p-1}}, \quad z \in U, \quad \zeta \in \bar{U}
$$

Then the function $F(z, \zeta)$ is analytic in $U \times \bar{U}$ and $F(0, \zeta)=1$.

From (2.9), we have

$$
z^{\alpha_{1}} G(z, \zeta)=\left(\alpha_{1}+p\right) \int_{0}^{z} t^{\alpha_{1}-1} f(t, \zeta) d t
$$

Differentiating both sides of (2.12) with respect to $z$, we get

$$
\left(\alpha_{1}+p\right) f(z, \zeta)=\alpha_{1} G(z, \zeta)+z G_{z}^{\prime}(z, \zeta)
$$

and

$$
\left(\alpha_{1}+p\right) H_{p}^{l, m}\left(\alpha_{1}\right) f(z, \zeta)=\alpha_{1} H_{p}^{l, m}\left(\alpha_{1}\right) G(z, \zeta)+z\left(H_{p}^{l, m}\left(\alpha_{1}\right) G(z, \zeta)\right)_{z}^{\prime} .
$$

So

$$
\begin{aligned}
\frac{\left(H_{p}^{l, m}\left(\alpha_{1}\right) f(z, \zeta)\right)_{z}^{\prime}}{p z^{p-1}}= & \frac{\left(\alpha_{1}+1\right)}{p\left(\alpha_{1}+p\right)} \frac{\left(H_{p}^{l, m}\left(\alpha_{1}\right) G(z, \zeta)\right)_{z}^{\prime}}{z^{p-1}} \\
& +\frac{1}{p\left(\alpha_{1}+p\right)} \frac{\left(H_{p}^{l, m}\left(\alpha_{1}\right) G(z, \zeta)\right)_{z}^{\prime \prime}}{z^{p-2}}
\end{aligned}
$$


From (2.11) and (2.13), we obtain

$$
F(z, \zeta)+\frac{1}{\alpha_{1}+p} z F_{z}^{\prime}(z, \zeta)=\frac{\left(H_{p}^{l, m}\left(\alpha_{1}\right) f(z, \zeta)\right)_{z}^{\prime}}{p z^{p-1}} .
$$

Using (2.14), (2.10) becomes

$$
F(z, \zeta)+\frac{1}{\alpha+2 p} z F_{z}^{\prime}(z, \zeta) \prec \prec q(z, \zeta)+\frac{1}{\alpha+2 p} z q_{z}^{\prime}(z, \zeta) .
$$

An application of Lemma 1.2 yields $F(z, \zeta) \prec \prec q(z, \zeta)$. By using (2.10), we obtain.

$$
\frac{\left(H_{p}^{l, m}\left(\alpha_{1}\right) G(z, \zeta)\right)_{z}^{\prime}}{p z^{p-1}} \prec \prec q(z, \zeta) .
$$

\section{References}

[1] B. C. Carlson and D. B. Shaffer, Starlike and prestarlike hypergeometric functions, SIAM J. Math Anal. 15 (1984), 737-745. https://doi.org/10.1137/0515057

[2] N. E. Cho, O. S. Kwon and H. M. Srivastava, Strong differential subordination and superordination for multivalently meromorphic functions involving the Liu-Srivastava operator, Integral Transforms Spec. Funct. 21(8) (2010), 589-601.

https://doi.org/10.1080/10652460903494751

[3] J. Dziok and H. M. Srivastava, Classes of analytic functions with the generalized hypergeometric function, Appl. Math. Comput. 103(1) (1999), 1-13.

https://doi.org/10.1016/S0096-3003(98)10042-5

[4] Ju. E. Hohlov, Operators and operations on the class of univalent functions, Izvestiya Vysshikh Uchebnykh Zavedenii. Matematika 10(197) (1978), 83-89.

[5] M. P. Jeyaraman and T. K. Suresh, Strong differential subordination and superordination of analytic functions, J. Math. Anal. Appl. 385(2) (2012), 854-864.

https://doi.org/10.1016/j.jmaa.2011.07.016

[6] J.-L. Liu, Strongly starlike functions associated with the Dziok-Srivastava operator, Tamkang J. Math. 35(1) (2004), 37-42.

[7] S. S. Miller and P. T. Mocanu, On some classes of first-order differential subordinations, Michigan Math. J. 32(2) (1985), 185-195. https://doi.org/10.1307/mmj/1029003185 
[8] S. S. Miller and P. T. Mocanu, Differential Subordinations: Theory and Applications, Series on Monographs and Textbooks in Pure and Applied Mathematics Vol. 225, Marcel Dekker Inc., New York and Basel, 2000.

[9] G. I. Oros and Gh. Oros, Strong differential subordination, Turk. J. Math. 33 (2009), 249257.

[10] S. Owa and H. M. Srivastava, Univalent and starlike generalized hypergeometric function, Canad. J. Math. 39(5) (1987), 1057-1077. https://doi.org/10.4153/CJM-1987-054-3

[11] S. Ruscheweyh, New criteria for univalent functions, Proc. Amer. Math. Soc. 49 (1975), 109-115. https://doi.org/10.1090/S0002-9939-1975-0367176-1

[12] A. K. Wanas and Alb Lupaş Alina, On a new strong differential subordinations and superordinations of analytic functions involving the generalized differential operator, Int. J. Pure Appl. Math. 116(3) (2017), 571-579.

[13] A. K. Wanas and A. H. Majeed, New strong differential subordinations and superordinations of symmetric analytic functions, Int. J. Math. Anal. 11(11) (2017), 543549. https://doi.org/10.12988/ijma.2017.7469

[14] A. K. Wanas and A. H. Majeed, Strong differential subordinations for higher-order derivatives of multivalent analytic functions defined by linear operator, Khayyam J. Math. 3(2) (2017), 160-171.

[15] A. K. Wanas and A. H. Majeed, Strong differential subordination and superordination of meromorphic multivalent quasi-convex functions, Kragujevac J. Math. 44(1) (2020), 2739. 